\title{
Chaotic Systems Synchronization Via High Order Observer Design
}

\author{
J. L. Mata-Machuca ${ }^{1}$, R. Martínez-Guerra ${ }^{1}$, R. Aguilar-López ${ }^{* 2}$ \\ ${ }^{1}$ Department of Automatic Control, CINVESTAV-IPN, \\ Mexico D.F., Mexico \\ ${ }^{2}$ Department of Biotechnology and Bioengineering, \\ CINVESTAV-IPN, Mexico D.F., Mexico \\ Phone (55) 57473733 Fax (55) 57473982 \\ jmata\{rguerra\}@ctrl.cinvestav.mx \\ *raguilar@cinvestav.mx
}

\begin{abstract}
In this paper, we consider the synchronization problem via a nonlinear observer design. A new exponential polynomial observer for a class of nonlinear oscillators is proposed, which is robust against output noises. A sufficient condition for synchronization is derived analytically with the help of the Lyapunov stability theory. The proposed technique has been applied to synchronize chaotic systems (Rikitake and Rössler systems) by numerical simulation.
\end{abstract}

Keywords: synchronization, polynomial observer, Lipschitz system, algebraic observability condition.

\section{RESUMEN}

En este trabajo se considera el problema de sincronización por medio del diseño de un observador no lineal. Se propone un nuevo observador polinomial exponencial para una clase de osciladores no lineales. La condición suficiente para lograr la sincronización es desarrollada analíticamente con la ayuda de la teoría de estabilidad de Lyapunov. La técnica propuesta ha sido aplicada para sincronizar sistemas caóticos (los sistemas de Rikitake y Rössler) empleando simulaciones numéricas.

\section{Introduction}

In the last years, the problem of synchronization of chaotic systems has received a great deal of attention among scientist in many fields [1], [2], [3], [4], [5]. In general, the synchronization research has been focused on two areas. The first one relates with the employ of state observers, where the main application is the synchronization of nonlinear oscillators [6], [7], [8], [9], [10]. The second one is the use of control laws, which allows achieving the synchronization with different structure and order between nonlinear oscillators [11], [12]. A particular interest is the connection between the observers for nonlinear systems and chaos synchronization, which is also known as master- slave configuration [15]; thus, the chaos synchronization problem can be regarded as an observer design procedure, where the coupling signal is viewed as output and the slave system is the observer [4], [9], [13]. In this configuration, the two coupled systems are identical and, therefore, identical synchronization occurs, which means that the difference of master and slave state vectors converges to zero for $t \rightarrow \infty$.
In this paper the synchronization scheme is proposed for a class of Lipschitz nonlinear systems. Many problems in engineering and other applications are globally Lipschitz, for instance the sinusoidal terms in robotics. Nonlinearities which are square or cubic in nature are not globally Lipschitz, however, they are locally so; moreover, when such functions occur in physical systems, they frequently have a saturation in their growth rate, making them globally Lipschitz functions [14]. Thus, this class of systems covered by this note is fairly general.

The main contribution of this paper consists in the solution of the synchronization problem via an exponential polynomial observer. In [14], [15], [16], existence conditions of the full-order observers for Lipschitz nonlinear systems were established. The main purpose in this work is to extend those results by showing that the conditions given in [16] also guarantee the existence of a full-order observer with a high-order correction term. The reason is very simple, as it is well known an extended 
Luenberger observer can be seen as a first order Taylor series around the observed state, therefore, to improve the estimation performance, a highorder term is now included in the observer structure.

The intention of choosing two examples as the Rössler and Rikitake systems is to clarify the proposed methodology; however, it is worth to mention that this technique can be applied to almost any chaotic synchronization problem.

\section{Exponential polynomial observer}

\section{A. Problem statement}

Consider the following nonlinear system:

$$
\begin{aligned}
& \dot{x}=f(x, u) \\
& y=C x
\end{aligned}
$$

Where $x \in \mathfrak{R}^{n}$ the vector of the state variables; $u \in \mathfrak{R}^{l}, \quad(l \leq n), \quad$ is the control input; $f(\circ): \Re^{n} \times \Re^{l} \rightarrow \Re^{l}, \quad$ is a nonlinear smooth vector function and Lipschitz in $x$ and uniformly bounded in $u ; y \in \mathfrak{R}$ is the vector of measured states, with $C \in \mathfrak{R}^{1 \times n}$.

Any nonlinear system of the form of Eq. (1a) can be expressed in the form of Eq. (1b) as long as $f(x, u)$ is differentiable with respect to $x$.

$$
\begin{gathered}
\dot{x}=A x+\Psi(x, u) \\
y=C x \quad, \quad x_{0}=x\left(t_{0}\right)
\end{gathered}
$$

In system (1b), $\Psi(x, u)$ is a nonlinear vector function which satisfies the Lipschitz condition with a Lipschitz constant $L$, i.e,

$$
\|\Psi(x, u)-\Psi(\hat{x}, u)\| \leq L\|x-\hat{x}\|
$$

B. A note on the Algebraic Observability Condition (AOC)

Before proposing the exponential polynomial observer, a definition concerning an algebraic observability condition is given (for more details see [17]).

Definition 1: Consider the system described by systems (1b), where $x \in \mathfrak{R}^{n}$. A state $x_{i}$, is said to be algebraically observable with respect to $\{u, y\}$ if it satisfies a differential polynomial in terms of $u$, $y$ and some of their time derivatives, i. e., $P\left(x_{i}, u, \dot{u}, \ldots, y, \dot{y}, \ldots\right)=0,1 \leq i \leq n$.

\section{Observer design}

We consider system (1b), the observer has the next form

$$
\begin{gathered}
\dot{\hat{x}}=A \hat{x}+\Psi(\hat{x}, u)+K_{1} C(x-\hat{x})+K_{2}[C(x-\hat{x})]^{m} \\
\hat{x}_{0}=\hat{x}\left(t_{0}\right)
\end{gathered}
$$

Throughout this paper, the following assumptions are considered:

Assumption 1: $m \in Z^{+}, m$ odd,$m>1$

Assumption 2: $K_{1}$ can be chosen such as the following Algebraic Riccati Equation (ARE) which has a symmetric positive-definite solution $P$ for some $\varepsilon>0$

$\left(A-K_{1} C\right)^{T} P+P\left(A-K_{1} C\right)+L^{2} P P+I+\varepsilon I=0$

Assumption 3: $K_{2}$ can be chosen such as the following relation which holds

$$
\lambda_{\min }\left(N+N^{T}\right) \geq 0, \text { with } N:=P K_{2} C .
$$

In (3), $\quad \hat{x} \in \mathfrak{R}^{n}, \quad K_{1}=\left[\begin{array}{llll}k_{1,1} & k_{2,1} & \ldots & k_{n, 1}\end{array}\right]^{T} \in \mathfrak{R}^{n}$ and $K_{2}=\left[\begin{array}{llll}k_{1,2} & k_{2,2} & \ldots & k_{n, 2}\end{array}\right]^{T} \in \mathfrak{R}^{n}$.

We analyze the observer error which is defined as $e=x-\hat{x}$. From (1b) and (3), the dynamics of the observer error $e=x-\hat{x}$ are given by

$\dot{e}=\left(A-K_{1} C\right) e-K_{2}[C e]^{m}+[\Psi(x, u)-\Psi(\hat{x}, u)]$ 
The following theorem proves observer convergence.

Theorem 1: For the nonlinear system (1b), suppose $x(t)$ exists for all $t \geq 0$ and the nonlinear vector function $\Psi(x, u)$ satisfies the Lipschitz condition (2). If a matrix $0<P=P^{T}$ and observer gains $K_{1}$ and $K_{2}$ can be found such that (3) is an observer for (1b), then the observer error converges to zero exponentially; that is, there exist constants $\kappa>0$ and $\lambda>0$ such that

$$
\|e(t)\| \leq \kappa\|e(0)\| \exp (-\lambda t)
$$

where $\quad \kappa=\sqrt{\frac{\beta}{\alpha}}, \quad \lambda=\frac{\varepsilon}{2 \beta}, \quad \alpha=\lambda_{\text {min }}(P), \quad$ and $\beta=\lambda_{\max }(P)$.

Proof: Consider the Lyapunov function candidate, $V=e^{T} P e$, where $0<P=P^{T}$ and satisfies Eq. (3b).

Its derivative is

$$
\begin{aligned}
\dot{V}= & \dot{e}^{T} P e+e^{T} P \dot{e} \\
= & e^{T}\left[\left(A-K_{1} C\right)^{T} P+P\left(A-K_{1} C\right)\right] \\
& -2(C e)^{m-1} e^{T} P K_{2} C e+2 e^{T} P[\Psi(x, u)-\Psi(\hat{x}, u)]
\end{aligned}
$$

In [14] the next inequality is presented based on (2) as a lemma which is useful for this proof,

$$
2 e^{T} P[\Psi(x, u)-\Psi(\hat{x}, u)] \leq L^{2} e^{T} P P e+e^{T} e
$$

From Rayleigh inequality [18], and taking into account inequality (3c), we have

$$
-2 e^{T} P K_{2} C e=-e^{T}\left(N+N^{T}\right) e \leq-\lambda_{\min }\left(N+N^{T}\right)\|e\|^{2}
$$

where $N:=P K_{2} C$.

Eq. (5) leads to

$$
\dot{V} \leq e^{T}\left[\left(A-K_{1} C\right)^{T} P+P\left(A-K_{1} C\right)\right] e
$$

$$
\begin{aligned}
& -(C e)^{m-1} \lambda_{\min }\left(N+N^{T}\right)\|e\|^{2}+L^{2} e^{T} P P e+e^{T} e \\
= & e^{T}\left[\left(A-K_{1} C\right)^{T} P+P\left(A-K_{1} C\right)+L^{2} P P+I\right] e \\
- & 2(C e)^{m-1} \lambda_{\min }\left(N+N^{T}\right)\|e\|^{2}
\end{aligned}
$$

From assumption 1, the second term in the right hand side of the inequality (5) always will be positive or zero,

$$
\begin{aligned}
\dot{V} & \leq e^{T}\left[\left(A-K_{1} C\right)^{T} P+P\left(A-K_{1} C\right)+L^{2} P P+I\right] e \\
& =-\varepsilon\|e\|^{2}
\end{aligned}
$$

We write the Lyapunov function as $V=\|e\|_{P}^{2}$, where $\quad \alpha\|e\|^{2} \leq V(e) \leq \beta\|e\|^{2}$, with $\quad \alpha=\lambda_{\text {min }}(P)$ $\in \mathfrak{R}^{+}$, and $\beta=\lambda_{\text {max }}(P) \in \mathfrak{R}^{+}$. Taking its derivative and replacing in inequality (6), we obtain

$$
\frac{d}{d t}\|e\|_{P} \leq-\frac{\varepsilon}{2 \beta}\|e\|_{P}
$$

Finally, we have the next result

$$
\begin{aligned}
& \|e\| \leq \kappa\|e(0)\| \exp (-\lambda t), \quad \text { where } \quad \kappa=\sqrt{\frac{\beta}{\alpha}}, \text { and } \\
& \lambda=\frac{\varepsilon}{2 \beta} \text {. }
\end{aligned}
$$

This implies that system (3) is an observer for system (1b) and the corresponding dynamics of the observer error (4) is exponentially stable.

\section{Application to sincronization of chaotic systems}

To illustrate the effectiveness of the obtained results, we give two applications to chaotic systems. The former is an application to the denominated Rössler system which presents a chaotic behavior and exhibits the simplest possible strange attractor. Originally, the Rössler system is credited to Otto Rössler, and it is said to be originated from work on chemical kinetics [21] and the second one is the so-called Rikitake system, a model which attempts to explain the reversal of the earth's magnetic field [22]. 


\section{A. Example 1: Rössler system}

We consider the popular nonlinear Rössler's System [19], which is described by

$$
\begin{aligned}
& \dot{x}_{1}=-\left(x_{2}+x_{3}\right) \\
& \dot{x}_{2}=x_{1}+a x_{2} \\
& \dot{x}_{3}=b+x_{3}\left(x_{1}-c\right) \\
& y=x_{1}
\end{aligned}
$$

It is well known that in a large neighborhood of $\{a=$ $b=0.2, c=5\}$ this system has a chaotic behavior.

Remark 1: It is not difficult to prove that system (7) is Lipschitz.

Before proposing the state observer, we prove the algebraic observability condition (see definition 1) for system (7). Replacing $y=x_{1}$ into system (7), we obtain

$$
\begin{aligned}
& \dot{y}=-x_{2}-x_{3} \\
& \dot{x}_{2}=y+a x_{2} \\
& \dot{x}_{3}=b+x_{3}(y-c)
\end{aligned}
$$

Taking the time derivative from Eq. (8)

$$
\ddot{y}=-\dot{x}_{2}-\dot{x}_{3}
$$

From Eq. (8), we get

$$
x_{3}=-\dot{y}-x_{2}
$$

Replacing Eqs. (9), (10) and (12) into Eq. (11)

$$
y-y \dot{y}+c \dot{y}+y-x_{2} y+(a+c) x_{2}+b=0
$$

In the same manner for $x_{3}$, we have from Eq. (8)

$$
x_{2}=-\dot{y}-x_{3}
$$

substituting Eqs. (9), (10) and (14) into Eq. (11)

$$
\ddot{y}-a \dot{y}+y+x_{3} y-(a+c) x_{3}+b=0
$$

Remark 2: From Eqs. (13) and (15), is clear that $x_{2}$ and $x_{3}$ are algebraically observable.

According to Theorem 1, we get the following system (slave system) for the observer

$$
\begin{aligned}
& \dot{\hat{x}}_{1}=-\left(\hat{x}_{2}+\hat{x}_{3}\right)+k_{1,1}\left(x_{1}-\hat{x}_{1}\right)+k_{1,2}\left(x_{1}-\hat{x}_{1}\right)^{m} \\
& \dot{\hat{x}}_{2}=\hat{x}_{1}+a \hat{x}_{2}+k_{2,1}\left(x_{1}-\hat{x}_{1}\right)+k_{2,2}\left(x_{1}-\hat{x}_{1}\right)^{m} \\
& \dot{\hat{x}}_{3}=b+\hat{x}_{3}\left(\hat{x}_{1}-c\right)+k_{3,1}\left(x_{1}-\hat{x}_{1}\right)+k_{3,2}\left(x_{1}-\hat{x}_{1}\right)^{m}
\end{aligned}
$$

We show some simulations for the Rössler system (7) and its observer given by system (16), we have taken for the parameter values $a=b=0.2, c=5$, $K_{1}=\left[\begin{array}{lll}k_{1,1} & k_{2,1} & k_{3,1}\end{array}\right]^{T}=\left[\begin{array}{lll}5 & -5 & 5\end{array}\right]^{T}, K_{2}=\left[\begin{array}{lll}k_{1,2} & k_{2,2} & k_{3,2}\end{array}\right]^{T}$ $=\left[\begin{array}{lll}10 & 10 & 10\end{array}\right]^{T}, m=3$. All simulation results in this paper were carried out with the help of Matlab 7.1 Software with Simulink 6.3 as the toolbox. The design of the exponential observer presented in this paper is based on the solution of the Riccati Equation which can be obtained by using the Matlab function ARE.

The performance index of the corresponding synchronization process is calculated as [20]

$$
J(t)=\frac{1}{t+0.001} \int_{0}^{t}\|e(t)\|_{Q_{0}}^{2} d \tau \quad, \quad Q_{0}=I
$$

where $e(t)$ denotes the estimation error.

Figures 1(a)-(c) show the convergence of the estimated states (slave system) to the real states (master system), without any noise in the system output. The initial conditions are $x_{1}=-0.5$, $x_{2}=0.5, x_{3}=4, \hat{x}_{1}=-4, \hat{x}_{2}=3, \hat{x}_{3}=-4$.

Figures 2(a)-(b) show the chaotic behavior of system (7) and the observer given by system (16), and also show the convergence of the state estimates to the real states, without any noise in the system output.

Furthermore, Figures 3(a)-(c) show the effect of noise in the estimation process. A white noise is added to the measurement $(\sigma=0.1, \pm 10 \%$ around the current value of the measured output). We can see that the exponential polynomial observer is robust against noisy measurement. 
Finally, Figure 4 illustrates the performance index for the corresponding estimation processes. It should be noted that the quadratic estimation error (performance index) is bounded and has a tendency to decrease.
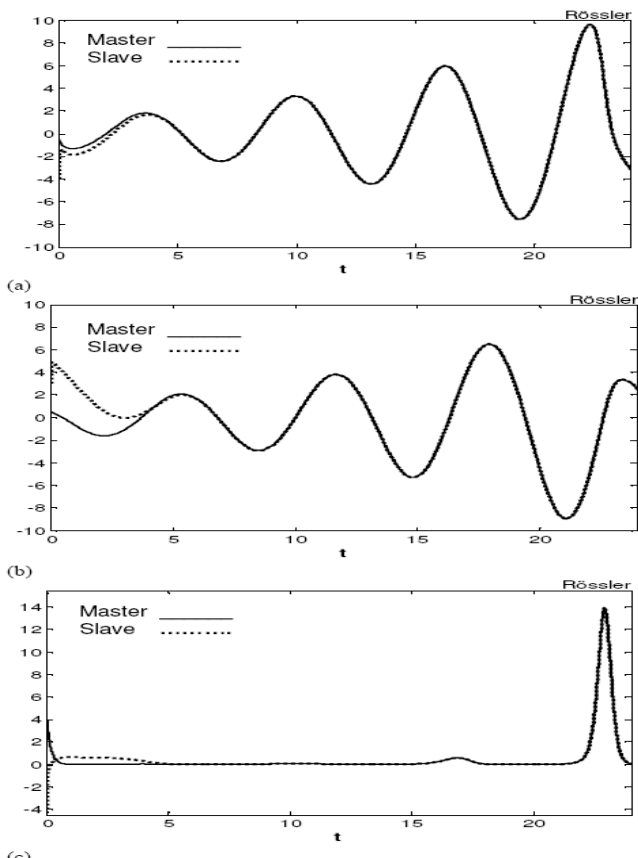

Figure 1. Synchronization between drive system (7) and response system (16), without any noise in the system output, (a)-signals $x_{1}$ and $\hat{x}_{1} ;(\mathrm{b})$-signals and $x_{2} ;$ (c)-signals $x_{3}$ and $\hat{x}_{3}$.
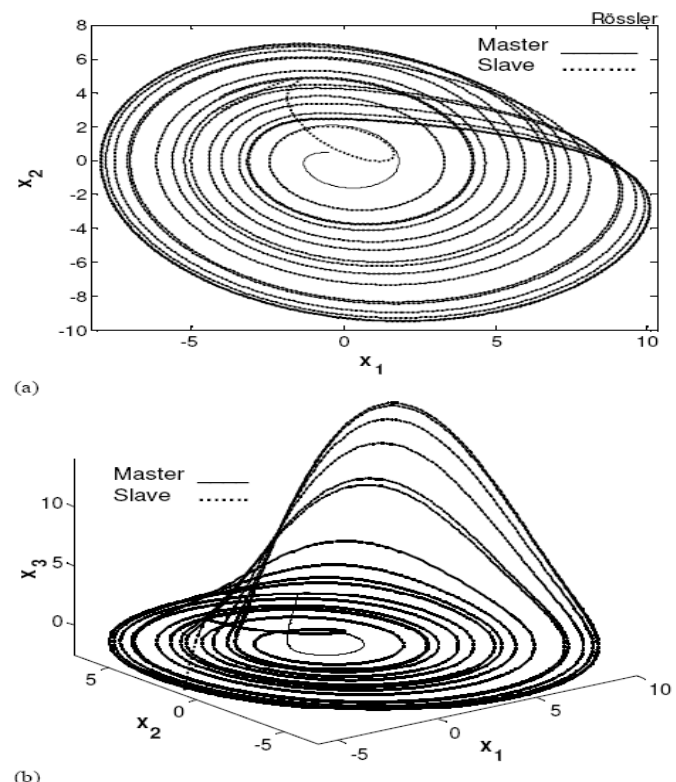

(b)

Figure 2. Chaotic behavior of drive system (7) and response system (16), without any noise in the system output, (a)- signals $x_{1}, x_{3}$ and $\hat{x}_{1}, \hat{x}_{3} ;(\mathrm{b})-$ signals $x_{1}, x_{2}, x_{3}$ and $\hat{x}_{1}, \hat{x}_{2}, \hat{x}_{3}$. 

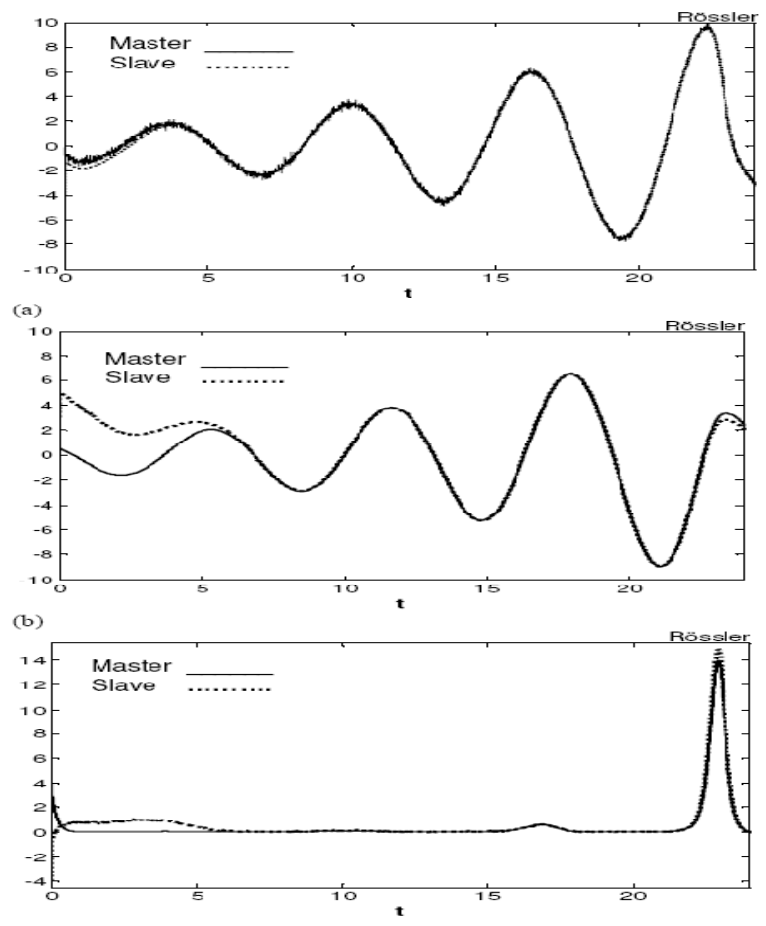

Figure 3. Synchronization between drive system (7) and response system (16), with white noise in the system output $(\sigma=0.1),(\mathrm{a})$ - signals $x_{1}$ and $\hat{x}_{1} ;(\mathrm{b})$ - signals $x_{2}$ and $\hat{x}_{2}$;

(c)- signals $x_{3}$ and $\hat{x}_{3}$.

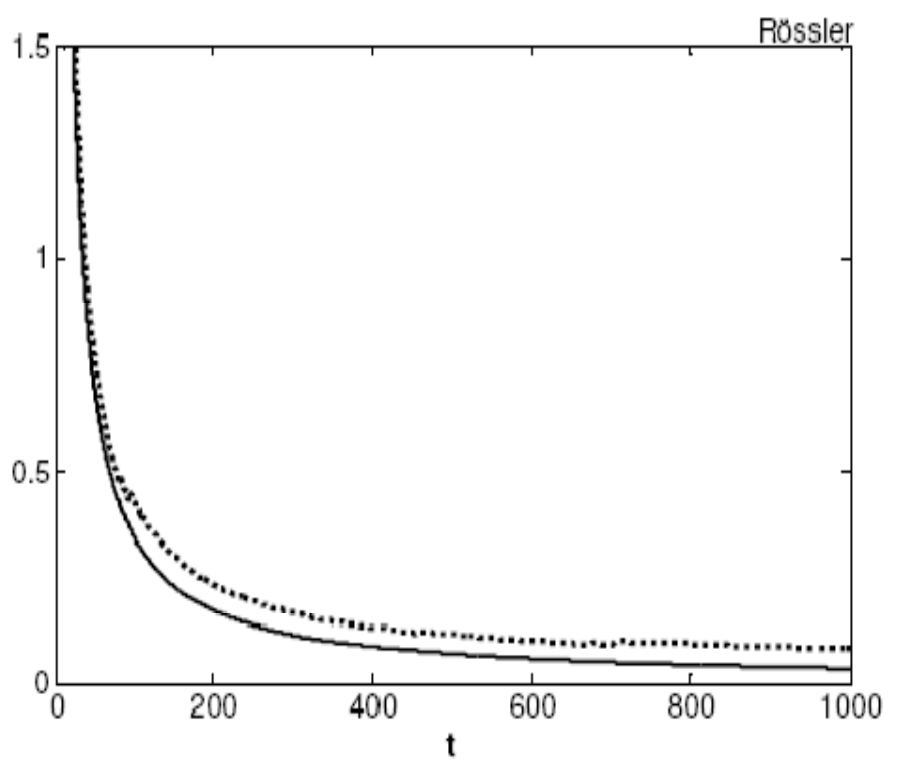

Figure 3. Quadratic estimation error, (a) - without any noise in the system output (solid line); (b) - with white noise $(\sigma=0.1)$ in the system output (dotted line). 


\section{B. Example 2: Rikitake oscillator}

This system describes the currents of two coupled dynamo disks [21]. The governing equations are

$$
\begin{aligned}
& \dot{x}_{1}=-\mu x_{1}+x_{2} x_{3} \\
& \dot{x}_{2}=-\mu x_{2}+\left(x_{3}-a\right) x_{1} \\
& \dot{x}_{3}=1-x_{2} x_{1} \\
& y=x_{1}
\end{aligned}
$$

here $a$ and $\mu$ are parameters which we will assume to be nonnegative.

Remark 3: It is not hard to see that the above system is Lipschitz.

Before proposing the exponential polynomial observer, we prove the algebraic observability condition (see definition 1) for system (17).

Replacing $y=x_{1}$ in system (17), we obtain:

$$
\begin{aligned}
& \dot{y}=-\mu \quad y+x_{2} x_{3} \\
& \dot{x}_{2}=-\mu x_{2}+\left(x_{3}-a\right) y \\
& \dot{x}_{3}=1-x_{2} y
\end{aligned}
$$

Taking the derivative with respect to time from Eq. (18), we have

$$
\ddot{y}=-\mu \dot{y}+\dot{x}_{2} x_{3}+x_{2} \dot{x}_{3}
$$

from Eq. (18), we get

$$
x_{3}=\frac{1}{x_{2}}[\dot{y}+\mu y]
$$

substituting Eqs. (19), (20) and (22) into Eq. (21), we obtain

$$
\begin{aligned}
0= & x_{2}^{4}-\frac{1}{y} x_{2}^{3}+\left[\frac{\ddot{y}}{y}+2 \mu \frac{\dot{y}}{y}+\mu^{2}\right] x_{2}^{2} \\
& +a[\dot{y}+\mu y] x_{2}-[\dot{y}+\mu y]^{2}
\end{aligned}
$$

In the same manner, for $x_{3}$ we have from Eq. (18)

$$
x_{2}=\frac{1}{x_{3}}[\dot{y}+\mu y]
$$

substituting Eqs. (19), (20) and (24) into Eq. (21)

$$
\begin{aligned}
0= & x_{3}^{4}-a x_{3}^{3}-\left(\frac{\ddot{y}}{y}+2 \mu \frac{\dot{y}}{y}+\mu^{2}\right) x_{3}^{2} \\
& +\left(\frac{\dot{y}}{y}+\mu\right) x_{3}-(\dot{y}+\mu y)^{2}
\end{aligned}
$$

Remark 4: From Eqs. (23) and (25), $x_{2}$ and $x_{3}$ are algebraically observable.

Going back to the original coordinate system, we get the following system (slave system) for the observer:

$$
\begin{aligned}
& \dot{\hat{x}}_{1}=-\mu \hat{x}_{1}+\hat{x}_{2} \hat{x}_{3}+k_{1,1}\left(x_{1}-\hat{x}_{1}\right)+k_{1,2}\left(x_{1}-\hat{x}_{1}\right)^{m} \\
& \dot{\hat{x}}_{2}=-\mu \hat{x}_{2}+\left(\hat{x}_{3}-a\right) \hat{x}_{1}+k_{2,1}\left(x_{1}-\hat{x}_{1}\right)+k_{2,2}\left(x_{1}-\hat{x}_{1}\right)^{m} \\
& \dot{\hat{x}}_{3}=1-\hat{x}_{1} \hat{x}_{2}+k_{3,1}\left(x_{1}-\hat{x}_{1}\right)+k_{3,2}\left(x_{1}-\hat{x}_{1}\right)^{m}
\end{aligned}
$$

Now, some numerical results for the Rikitake system (17) and its observer given by system (26) are presented. System (17) is chaotic with the set of parameter values $\mu=1, a=0.375$.

We have chosen the parameter values for systems (17) and (26) as $\mu=1, a=0.375, m=3$,

$$
\begin{aligned}
K_{1} & =\left[\begin{array}{lll}
k_{1,1} & k_{2,1} & k_{3,1}
\end{array}\right]^{T}=\left[\begin{array}{lll}
2 & 2 & 2
\end{array}\right]^{T}, \\
K_{2} & =\left[\begin{array}{lll}
k_{1,2} & k_{2,2} & k_{3,2}
\end{array}\right]^{T}=\left[\begin{array}{lll}
3 & 3 & 3
\end{array}\right]^{T} .
\end{aligned}
$$

Figures 5(a)-(c) show the convergence of the estimated states (slave system) to the real states (master system), without any noise in the system output. The initial conditions are $x_{1}=-1, x_{2}=0.5$, $x_{3}=4, \hat{x}_{1}=-4, \hat{x}_{2}=-1, \hat{x}_{3}=2$.

Figures 6(a)-(c) show the chaotic behavior of the master system (17) and the slave system (26) and also show the convergence of the estimated states (slave system) to the real states (master system) without any noise in the system output. 


\section{Chaotic Systems Synchronization Via High Order Observer Design, J. L. Mata-Machuca et al., 57-68}

Figures 7(a)-(c) show the estimated states with the presence of noise in the system output (white noise with $\sigma=0.1, \pm 10 \%$ around the current value of the system output). It should be noted that the proposed observer is robust against noisy measurements.

Figure 8 illustrates the performance index for the corresponding synchronization process without any noise in the system output and with noise in the system output (white noise with $\sigma=0.1, \pm 10 \%$ around the current value of the system output). In both cases, the corresponding performance index has a tendency to decrease.

\section{Conclusion}

In this paper, we have designed a new exponential polynomial observer (high order polynomial type) for a class of nonlinear oscillators to attack the synchronization problem. Also, we have proven the exponential stability of the resulting state estimation error and by means of simple algebraic manipulations we construct the observer (slave system). Finally, we have presented some simulations to illustrate the effectiveness of the suggested approach, which shows some robustness properties against noisy measurements.
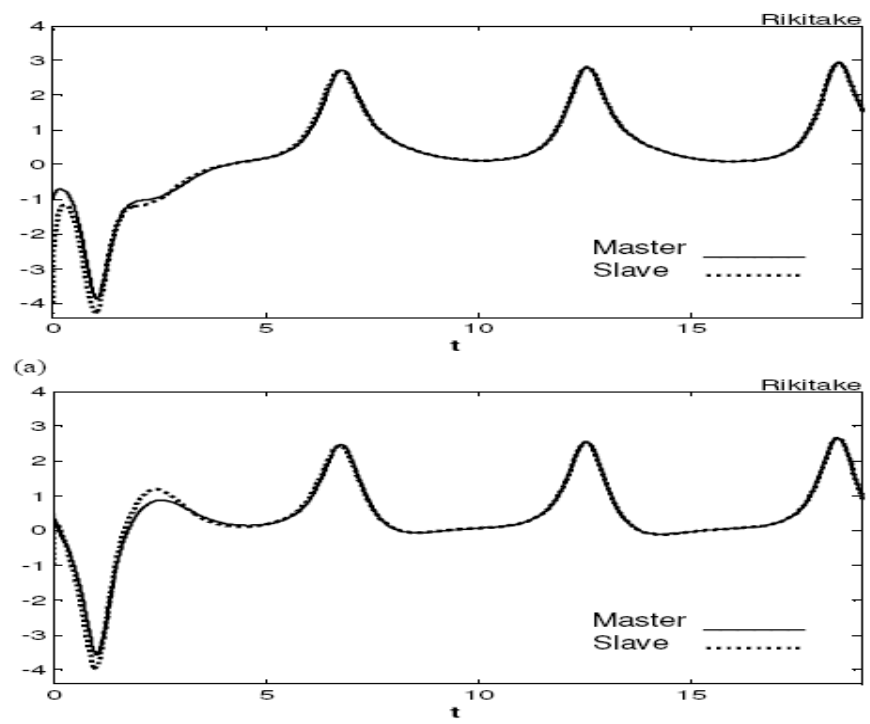

(b)

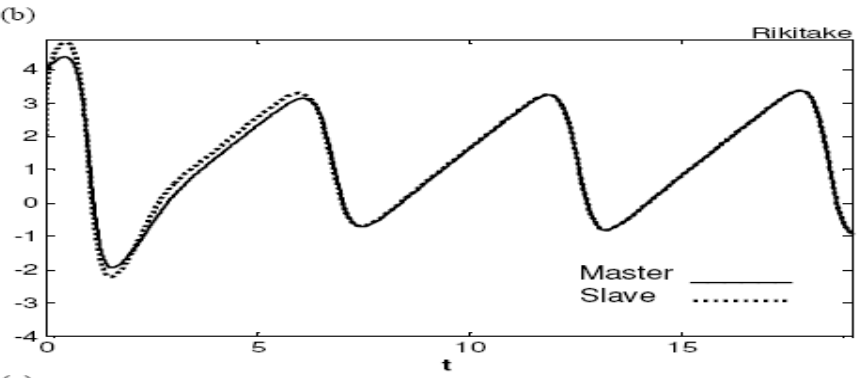

(c)

Figure 5. Synchronization between drive system (17) and response system (26), without any noise in the system output, (a) - signals $x_{1}$ and $\hat{x}_{1}$;

(b)- signals $x_{2}$ and $\hat{x}_{2} ;(\mathrm{c})$ - signals $x_{3}$ and $\hat{x}_{3}$. 

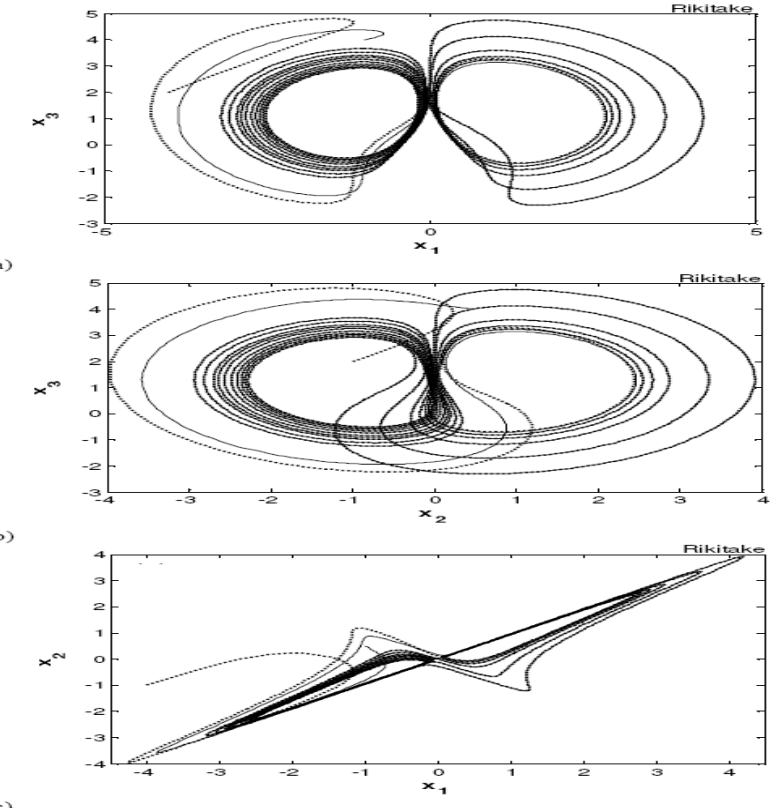

Figure 6. Chaotic behavior of drive system (17) and response system (26), without any noise in the system output, (a) - signals $x_{1}, x_{3}$ and $\hat{x}_{1}, \hat{x}_{3}$;

(b) - signals $x_{2}, x_{3}$ and $\hat{x}_{2}, \hat{x}_{3} ;(\mathrm{c})$ - signals $x_{1}, x_{2}$ and $\hat{x}_{1}, \hat{x}_{2}$.
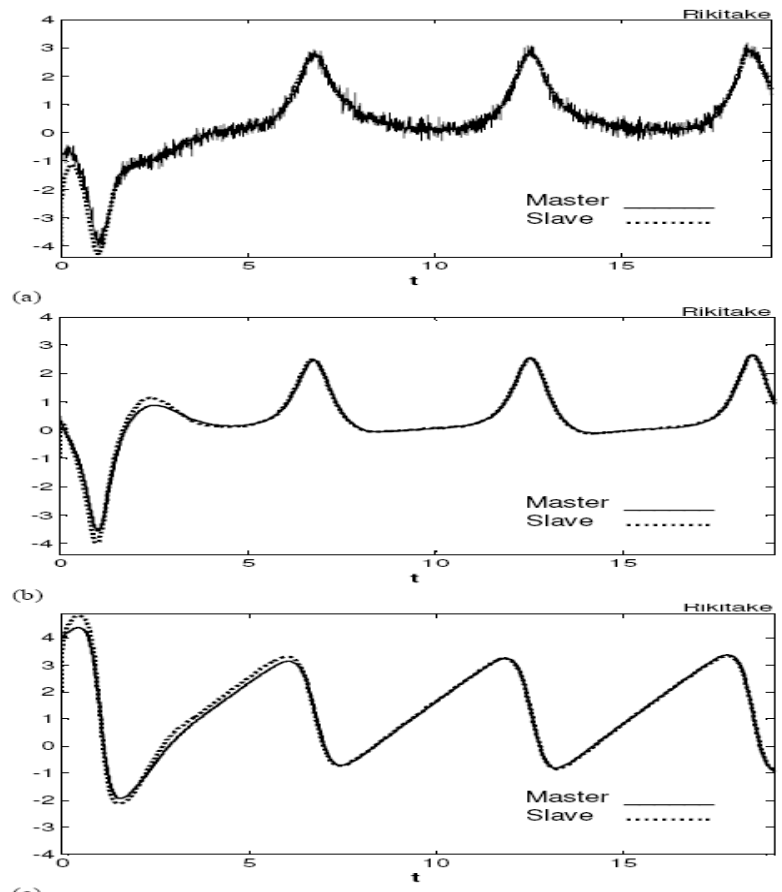

Figure 7. Synchronization between drive system (17) and response system (26), with white noise in the system output $(\sigma=0.1),(a)-$ signals $x_{1}$ and $\hat{x}_{1}$;

(b) - signals $x_{2}$ and $\hat{x}_{2} ;$ (c)-signals $x_{3}$ and $\hat{x}_{3}$. 


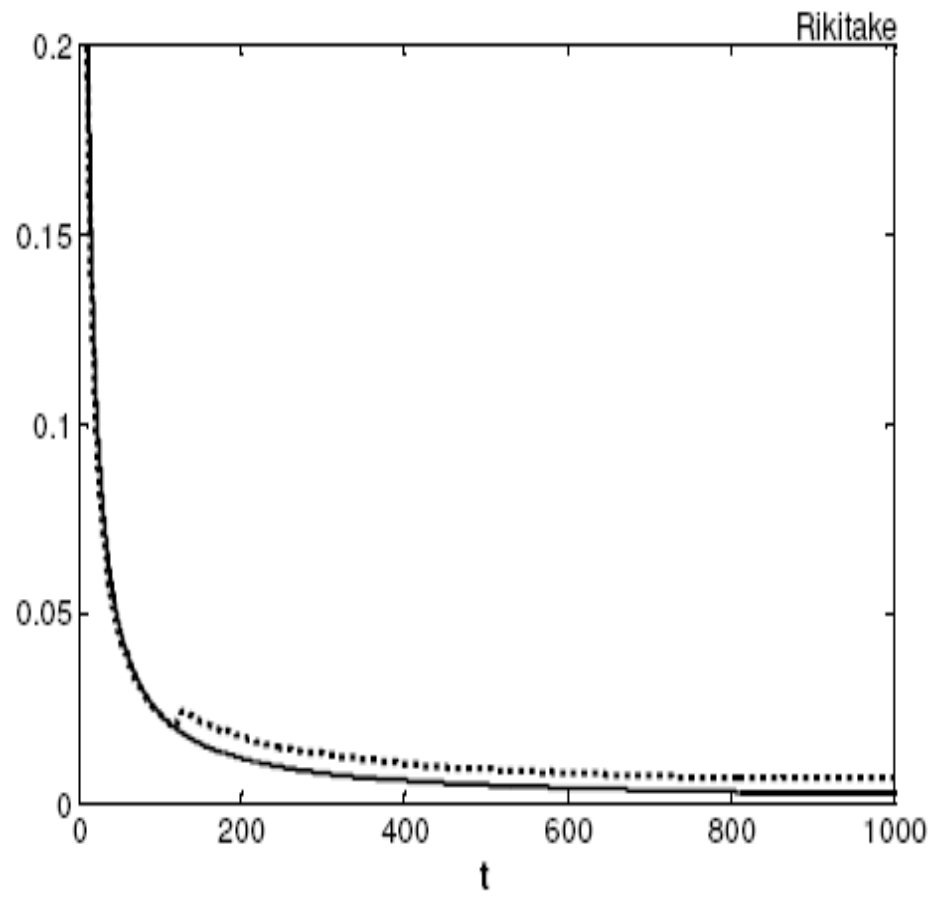

Figure 8. Performance index, (a) - without any noise in the system output (solid line); (b) - with white noise $(\sigma=0.1)$

in the system output (dotted line). 


\section{References}

[1] A. Fradkov. Cybernetical physics: from control of chaos to quantum control. Berlin: Springer, 2007.

[2] M. Chen, D. Zhou and Y. Shang, "A sliding mode observer based secure communication scheme," Chaos, Solitons Fractals, vol. 25, pp. 573-8, 2005.

[3] M. Feki, "Observer-based exact synchronization of ideal and mismatched chaotic systems," Physics Letters A, vol. 309, pp. 53-60, 2003.

[4] O. Morgül and M. Feki, "A chaotic masking scheme by using synchronized chaotic systems," Physics Letter A, vol. 251, pp. 169-176, 1999.

[5] R. Guo, "A simple adaptive controller for chaos and hyperchaos synchronization," Physics Letters A, vol. 372, pp. 5593-5597, 2008.

[6] R. Martínez-Guerra, W. Yu and E. Cisneros-Saldaña, "A new model-free sliding observer to synchronization problem," Chaos Solitons Fractals, vol. 36, pp. 1141-1156, 2008.

[7] R. Aguilar-Lopez and R. Martínez-Guerra, "Synchronization of a Class of Chaotic signals via robust observer design," Chaos, Solitons Fractals, vol. 37, pp. 581-587, 2008.

[8] C. Hua and X. Guan, "Synchronization of chaotic systems based on PI observer design," Physics Letters A, vol. 334, pp. 382-389, 2005.

[9] R. Martínez-Guerra, J. Cruz, R. Gonzalez, R. Aguilar, "A new reduced-order Observer design for the synchronization of Lorenz systems," Chaos, Solitons Fractals, vol. 28, pp. 511-7, 2006.

[10] O. Morgül and E. Solak, "Observed based synchronization of chaotic systems," Phys. Rev. E, vol. 54, pp. 4803-4811, 1996.

[11] R. Femat and G. Solís-Perales, Robust synchronization of chaotic systems via feedback, Springer Verlag, 2008.

[12] F. Wang and C. Liu, "A new criterion for chaos and hyperchaos synchronization using linear feedback control," Physics Letters A, vol. 360, pp. 274-278, 2006.

[13] L. Pecora, T. Caroll, "Synchronization in chaotic systems," Phys. Rev. Lett., vol. 64, pp. 821-4, 1990.
[14] S. Raghavan and J. Hedrick, "Observer design for a class of nonlinear systems," Int Journal Control, vol. 59, pp. 515-528, 1994.

[15] F. Thau, "Observing the state of non-linear dynamic systems," Int Journal Control, vol. 17, pp. 471-479, 1973.

[16] R. Rajamani, "Observers for Lipschitz nonlinear systems," IEEE Trans. Aut. Control, vol. 43, no.3, pp. 397-401, 1998.

[17] S. Diop and R. Martínez-Guerra, "An algebraic and data derivative information approach to nonlinear system diagnosis," in Proceedings of the European Control Conference (ECC), Porto, Portugal, 2001, pp. 23342339.

[18] R. Horn, C. Johnson, Matrix analysis, Cambridge University Press, New York, 1985, pp. 176.

[19] O. Rössler, "An Equation for Continuous Chaos," Phys. Lett., vol. 57 A, pp. 397-398, 1976.

[20] R. Martínez-Guerra, A. Poznyak and V. Díaz, "Robustness of high-gain observers for closed-loop nonlinear systems: theoretical study and robotics control application," Int Journal Systems Science, vol. 31, pp. 1519-1529, 2000.

[21] T. Rikitake, "Oscillations of a system of disk dynamos". Proc Cambridge Philos Soc, vol. 54, pp. 89105, 1958. 


\section{Authors' Biographies}

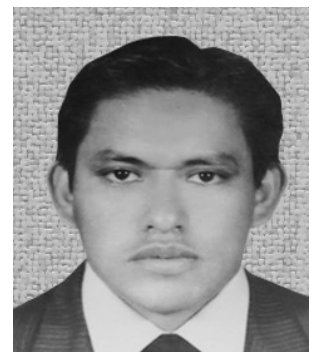

\section{Juan L. MATA- MACHUCA}

Juan L. Mata was born in 1983 in Veracruz, Mexico. He graduated from Universidad Veracruzana (University of Veracruz), UV, in mechanicalelectrical engineering in 2006. He obtained his M.Sc. degree from the Centro de Investigación y de Estudios Avanzados (Center for Research and Advanced studies) of the Instituto Politécnico Nacional (National Polytechnic Institute), CINVESTAV-IPN, Department of Automatic Control, in 2009, where he is currently enrolled as a PhD student. His research interests are chaos synchronization, monitoring of nonlinear dynamical systems, nonlinear observers, fault diagnosis, feedback control and fractional systems.

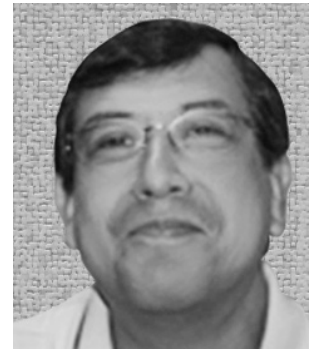

\section{Rafael MARTÍNEZ-GUERRA}

Rafael Martínez-Guerra was born in Mexico City in 1959, He earns his Ph. D. degree from Universidad Autonóma Metropolitana in 1996. Currently, he is a researcher at the Automatic Control Department of the Centro de Investigación y de Estudios Avanzados of the Instituto Politécnico Nacional and member of the National System of Researchers since 1992 (level II, currently). He is author and co-author of more than 50 papers in international journals and more than 90 contributions in international conferences with proceedings. His main research interests are in the field of nonlinear systems, differential geometric and differential algebraic methods, nonlinear observers, fault detection problems and chaos.

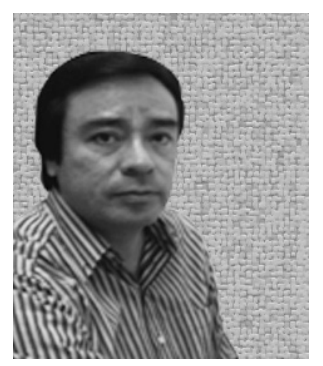

\section{Ricardo AGUILAR-LÓPEZ}

Ricardo Aguilar-López was born in Mexico City in 1964. He earns his B Sc, M Sc and Ph D degrees from Universidad Autónoma Metropolitana in 1989, 1993 and 1998, respectively; all of them in chemical engineering. He was a postdoctoral fellow at Instituto Mexicano del Petróleo (2000) besides he earns a Ph D in automatic control from CINVESTAV-IPN (2003). He works in robust observer design for nonlinear systems and process control. He is author and co-author of 88 papers published in international journals. Currently, he is a researcher at the Biotechnology and Bioengineering Department of the Centro de Investigación y de Estudios Avanzados of the Instituto Politécnico Nacional and member of the National System of Researchers since 1998, (currently level II). 